\title{
Towards Design Recommendations for Training of Security Critical Agents in Mixed Reality Environments
}

\author{
Jean-Louis Huynen \\ LIST \\ L-4422 Belvaux - LU \\ jeanlouis.huynen@list.lu
}

\author{
Rod McCall \\ LIST \\ L-4422 Belvaux - LU \\ roderick.mccall@list.lu
}

\author{
Mike Griffin \\ LIST \\ L-4422 Belvaux - LU \\ mike.griffin@augmented-solutions.ltd
}

\begin{abstract}
This paper presents an overview of a requirements capture process and identified design challenges for a Mixed Reality (MR) platform which is designed to be used by security critical agents at operational command level. The platform aims to support a wide range of scenarios ranging from firearms training to chemical and nuclear incidents. The paper identifies a number of key design issues which are required to provide effective training across the range of scenarios discussed.
\end{abstract}

Augmented Reality. User studies. Ethical issues.

\section{INTRODUCTION}

Security problems are increasing across the globe with chemical substances being released on the streets of Salisbury through to the tragic events in France. These and the many other events call for Security Critical Agents (SCAs) to undergo training that was until now reserved for special intervention units and specialised first responder teams. The rationale for this trend in training of SCAs is twofold, (1) most attacks are unsophisticated attacks on softtargets (Europol, 2016), and (2) ISIS and other terror groups have a strong interest in obtaining chemical, chemical, biological, radiological and nuclear materials (Bureau of Counterterrorism. United States Department of State, 2016).

SCAs need an ever growing range of competencies, knowledge and practical experience to cope with increasing problems. The diversity and often relative rarity of situations, which SCAs need to be prepared for, creates unprecedented challenges for effective SCA training. In addition, due to an increasing visibility of emergency services owing to social media and the ease with which photos and videos are shared, societal pressures are imposing more expectations from technology, more accountability from emergency services, and less tolerance for errors.

All these events require that national authorities in particular the police and security agencies can train effectively. However, such training approaches are often expensive, labour intensive and can require the use of live risks (e.g. radiation sources). In contrast the TARGET project aims to make training more cost effective through the combination of augmented reality and tangible interface objects (TARGET Consortium, 2018).

This paper outlines the design process for a multiscenario training system which uses a combination of mixed reality (virtual and real) and geospatial tools. It describes the requirements capture process undertaken and some of the design challenges which are unique within SCA training. Following on from this it identifies early guidelines which can be used to inform the design of such systems. The primary focus will be on mixed reality (virtual and augmented) requirements capture and design issues.

\section{BACKGROUND AND STATE OF THE ART}

\subsection{Current Platforms and Systems}

At present, law enforcement and rescue agencies train their staff through three distinct modalities: physical simulations, multimedia simulations, and desktop-based simulations. Physical simulations are the best to train as close as possible to real conditions, and can also be used as mission rehearsal exercises and to improve agent's readiness. Using real resources, physical simulations are expensive, mobilise resources that could be spent elsewhere for the time of the training, and may put these resources at risk. Multimedia and desktop-based simulations don't suffer from these 
drawbacks but instead lack of fidelity, in particular equipment fidelity (Knerr, 2007). This kind of training (e.g. Command Post Exercise, Tactical Exercise Without Troops, etc.) can be well-suited for strategic, and tactical-level operator training but clearly lack simulation fidelity for operation-level operators.

Firearm training simulators (for instance VirTra V-ST Pro) address this problem by providing operators with real equipment and allowing them to shoot simunitions on videos beamed on a screen (French police also use a system called EVA in which trainees fire live ammunitions). While these simulations are less expensive than physical simulations, are photo-realistic and offer hands-on training, they do not allow for real operator movements.

\subsection{Command Levels}

Three levels of command are supported within the system. These are: operational, tactical and strategic. Operation level can be thought of as boots on the ground e.g. firearms officers who have to undertake relatively short term, high stress tasks with potentially immediate outcomes. Tactical level command relates typically to short to medium term decision making tasks, this command level is normally located not too far away from the scene of the event (e.g. in a mobile command post/van). Strategic command is the highest level and focuses on longer term impacts and decisions. This level can include the highest level of Police Commander. Although the timeline is longer the impacts are potentially larger than at lower levels of command. The scenarios described in this paper focus on operational level exercises and command level.

\subsection{Mixed Reality}

Mixed Reality (MR) refers to a range of technologies including Augmented Reality (AR), Augmented Virtuality (AVR) and Virtual Reality (VR) see (Milgram \& Kishino, 1994).

\subsection{Blended Spaces, Presence and Flow}

The objective of the platform is that training could take place in a variety of locations for example in a shop, lab or office. As such the aim is to create a blended space which fuses the real world location and virtual (Benyon, Mival, \& Ayran, 2012) to create a new experience geared towards the improvement of real world performance. Related to his are topics such as flow (Csíkszentmihályi, 1990) and presence (IJsselsteijn, Riva, \& Davide, 2003). Flow is when a person is within a mental state that they feel completely immersed, involved and perhaps also a sense of enjoyment in the activity. Although the latter concept is not so relevant to the domain of SCA training. The resulting sense of flow should distort their sense of space and time. From the perspective of virtual environments, presence is when a person feels physically or socially presence (Lombard \& Ditton, 1997), the concept of co-presence will not be discussed here as it is not relevant for this project. A person is physically present in a virtual environment when they are not aware of the surrounding real world space. Social presence is when a person feels together with either another real or virtual person in the virtual experience. Virtual and augmented realities differ in the nature of the sense of presence that they provide. Therefore, while presence measures are relevant they need to be considered in the context of creating blended spaces or experiences.

There is a debate within the presence community as to the precise impacts of form, content and user characteristics in determining a sense of presence. Lombard and Ditton (Lombard \& Ditton, 1997) argue that all three have a role. Form focusses on aspects such as number and quality of sensory inputs (smell, auditory, visual) and obtrusiveness of the medium being used (e.g. a cumbersome headset). Content variables include aspects such as the nature of the task or activity and narrative etc. While the user characteristics include aspects such as the users willingness to suspend disbelief, prior knowledge or experience. Within a security training context all three elements are likely to play a part, for example the narrative will come from the training objectives, plan and scenario with behaviour being shaped by user characteristics which they may exhibit in real situations. Also form aspects such as believable characters are important.

\section{PROJECT OVERVIEW}

The TARGET project consists of six quite different training scenarios, however all are being developed on top of a common base with the aim of allowing organisations to decide which technologies and content aspects to use. For example, they can choose to use 2D geospatial environment to control the movement of police assets and see this rendered in real time in virtual reality, or chose to run an entirely augmented reality tactical firearms training exercise. This flexibility means that the platform can support from operational (ground level actions, such as a police firearms) through tactical and strategic high level command. In this paper we focus on three augmented reality scenarios as they are similar in terms of technologies and command level.

A critical aspect of the project is the inclusion of a serious games or gamification (Deterding, Khaled, Nacke, \& Dixon, 2011) approach into the design of the training scenarios. Such approaches are used to persuade users to change their actions, with the aim of improving training outcomes and real world performances. 


\subsection{Scenarios}

\subsubsection{CBRN Incidents}

CBRN (chemical, biological, radiological and nuclear). This scenario consists of using augmented reality and real world objects to simulate a clandestine CBRN laboratory using simulated detection devices and CBRN sources. The aim is to let teams train in the detection, identification and ultimately the extraction of dangerous materials while guaranteeing the involved operators' safety. Augmented reality allows for a safer training (than using simulant) and importantly can also be used to display contamination or dispersal of chemicals etc. which cannot normally be seen.

\subsubsection{Tactical Firearms Vignettes}

A police team composed by first responders and later by a special intervention group has to safely extract a hostage from an armed group inside a building, again ensuring that innocent people are not harmed. Augmented reality is used alongside physical objects to ensure that the scenario is kept as realistic as possible.

\subsubsection{Arrest of a Suspect after a Car Crash}

Two police officers (first responders) respond to a car crash. A criminal exits from the vehicle. Trainees must respond appropriately. This scenario uses a mix of real objects (a real police car) and AR for other elements (e.g. characters).

\section{UNDERSTANDING THE PROBLEM}

In order to understand the needs of the organisations, we performed a Training Needs Analysis (TNA). The main objectives of the TNA are (1) to obtain system's requirements, from a user's perspectives, and (2) to determine how the system's success will be evaluated. As a result, TNA is used to identify the gap between participants' current training and their training requirements. It involves determining whether training will provide the skills and procedures that need to be delivered operationally. Developing a fitting MR scenario for training of SCAs is challenging because there are organisational, technical, security, and ethical issues:

(i) A training system for SCA has to include organisation's corporate mission into the training design, and may encompass the full chain of command. Information about the strategic, tactical, and operational personnel training needs, as well as the requirements for individual competence have to be captured.

(ii) MR is a new technology whose potential is possibly hard to imagine for end-users. Training system may have to be fed data collected by legacy systems used in operations.

(iii) Due to the security nature of SCAs, some operational data can be too sensitive to be acknowledged by the training designers,

(iv) Finally, ethical issues, need to be closely considered throughout the development of MR training of SCA. We need in particular to ascertain that the training delivered won't foster adverse events.

Designing systems that solve complex-real-world problems such as the training of SCAs requires engagement with end-users (Checkland \& Poulter, 2006). In this section, we show how we performed Contextual Inquiries within our TNA in order to better align our MR training scenarios with the real training needs of SCAs and their hosting organisations. We follow this by discussing our first experience feedback and the challenges we identified.

\subsection{Methods}

\subsubsection{Training Needs Analysis}

First, TNA is used to identify what are the skills needed for each level of command. The relevant performance criteria, skills, and knowledge required are derived from the organisation's individual command level profiles.

\subsubsection{Contextual Inquiry}

To ascertain the end user requirements for a training scenario, part of the Contextual Inquiry $(\mathrm{Cl})$ and design process (Beyer \& Holtzblatt, 1998) (Holtzblatt \& Beyer, 1999) was used alongside the wider TNA approach. It is a methodology designed to derive requirements, and subsequently the overall system, from an embedded analysis of how people currently work. The emphasis is on observation and understanding of a work practice within a given context rather than starting from an underlying technological perspective and enforcing that upon the target group.

The $\mathrm{Cl}$ process involved:

(i) Visiting the end user responsible for the operational delivery of the training,

(ii) Observing and recording of similar exercises (or live play and simulations) to the planned training,

(iii) Interviewing with instructors and participants: structured debriefs are with 12 to 15 attendees who belong to the training or operational staff, and span the different level of command.

The results were then used as a basis for design of the training scenarios and technologies. 


\subsubsection{Ethics and Informed Consent}

All trials followed an ethics policy, with participants provided with an informed consent form, briefings (verbal and in written form) and a debriefing. They were informed that they could stop participating at any time and could request that their data was removed. Each participant also received a trials feedback form where they could highlight any issues they had during the study.

\section{CHALLENGES AND EXPERIENCE FEEDBACK}

\subsection{Scenario Design Issues}

All the scenarios are designed to prepare SCAs to take wicked decisions (in the sense that there is no right answer, only a better or worse solution (Webber \& Rittel, 1973)). Below we provide a list of common stress factors that the trainees have to handle in every scenario, which we believe represent the key design concepts and challenges for such systems:

(i) Regarding available information and the unfolding situation:

- Poor information regarding the threat,

- novelty of the environment and the situation,

- perception and communication difficulties, lack of sources of information,

- contradictory information,

- frightened or agitated members of the public

- direct and immediate threat to the public,

- ambiguous behaviours,

- people not obliging to requests.

(ii) Regarding decision-making:

- Lack of time to consider every consequence of actions,

- lack of situational awareness, or biases in how information is selected and used for taking decisions: in-attentional blindness (Mack \& Irvin, 1998) and the "tunnel effect".

(iii) Regarding consequences:

- Self-preservation and harm done to people,

- damage to property,

- destruction of evidence,

- ethical and legal implications,

- information communicated to the public.

\subsection{Ethical Constraints}

As noted during the visits, SCAs adhere to very high ethical practices, for example avoiding stereotyping, and the overarching concern is to preserve life. Related to this, standard operating procedures would normally dictate that all members of the public at location are assessed for their risk before being categorised as a criminal or a victim. This is standard practice and can often be observed in news reports concerning school shooter incidents, where all pupils have their hands up while being escorted out of a building.

Further problems also arise when game-like elements are added which may condition behaviour in undesirable ways in the longer term, even if during training the results are desirable. Furthermore, aspects which can be seen as beneficial or a desirable property of such a design (e.g., tunnelling, suggestion, or reduction) may increase the risks to the trainees or members of the public during real events. For example, tunnelling implies a focus on specific set of activities, which in turn may increase tunnel vision - which is not desirable in this context. Additionally, trainees may become too reliant on suggestions during training or become too used to responding to simplified tasks. Therefore, care has to be taken to avoid these issues which are often seen as key strategies to influencing behaviour (Fogg, 2003).

\section{CONCLUSION}

The collection of training needs for the design of a operational level MR training system for SCA lead us to inquire in detail into the training delivered by several Law Enforcement Agencies. MR training has the potential (in addition to teaching various skills available by other training means) to teach trainees to take hard decisions and to improve task automation when operating under various stress factors. Indeed, in addition to decision making, MR training lets trainees make mistakes, slips and lapses - like a mission rehearsal would do. However, it significantly improves on mission rehearsal approaches as it moves beyond simply pressing a button to simulate a police officer or terrorist getting out of a car. Getting out of a car with a protective jacket on and loaded weapons while being fired at is unsurprisingly a very stressful and perhaps error-prone process therefore a training environment which approximates this as closely as possible is required. The paper has also identified potential ethical challenges when employing gamification and related techniques to this area.

\section{ACKNOWLEDGEMENTS}

This work has been carried out within the framework of the EU H2020 TARGET project (TARGET Consortium, 2018). The project has received funding from the European Union's Horizon 
2020 research and innovation programme under grant agreement No 653350. See details on the TARGET project at (TARGET Consortium, 2018).

\section{REFERENCES}

Benyon, D., Mival, O., \& Ayran, S. (2012). Designing blended spaces. Proceedings of the 26th Annual BCS Interaction Specialist Group Conference on People and Computers. (pp. 398-403). Birmingham, United Kingdom: British Computer Society.

Beyer, H., \& Holtzblatt, K. (1998). Contextual design: Defining customer-centered systems. Morgan Kaufmann Publishers.

Bureau of Counterterrorism. United States Department of State. (2016). Country Reports on Terrorism 2016. United States Department of State Publication.

Checkland, P., \& Poulter, J. (2006). Learning for action: a short definitive account of soft systems methodology and its use, for practitioners, teachers and students. John Wiley and Sons Ltd.

Csíkszentmihályi, M. (1990). Flow: The psychology of optimal experience. New York: Harper and Row.

Dahlstrom, N., Dekker, S., ven Winsen, R., \& Nyce, J. (2009). Fidelity and validity of simulator training. Theoretical Issues in Ergonomics Science, 10(4), 305-314.

Deterding, S., Khaled, R., Nacke, L. E., \& Dixon, D. (2011). Gamification: Toward a definition. In CHI 2011 gamification workshop proceedings (Vol. 12). Vancouver BC, Canada.

Europol. (2016). Changes in modus operandi of Islamic State terrorist attacks. The Hague: Europol Public Information.

Fogg, B. J. (2003). Motivating, Influencing, and Persuading Users. In J. Jacko, \& A. Sears, Motivating, Influencing, and Persuading Users (pp. 358-370). Hillsdale, NJ, USA: L. Erlbaum Associates Inc.
Holtzblatt, K., \& Beyer, H. (1999). Contextual design. $\mathrm{CHI}$ '99 extended abstracts on Human factors in computing systems. Pittsburgh, Pennsylvania: ACM.

IJsselsteijn, W. A., Riva, G., \& Davide, F. (2003). Being There: Concepts, effects and measurement of user presence in synthetic environments. Amsterdam: los Press.

Knerr, B. W. (2007). Immersive Simulation Training for the Dismounted Soldier. Orlando, Florida, USA: US Department of Defense.

Lombard, M., \& Ditton, T. (1997). At the Heart of It All: The Concept of Presence. Journal of Computer-Mediated Communication, 3(2).

Mack, A., \& Irvin, R. (1998). Inattentional Blindness (Vol. 33). Cambridge: MA: MIT press.

Milgram, P., \& Kishino, F. (1994). A taxonomy of mixed reality visual displays. IEICE TRANSACTIONS on Information and Systems, 77(12), 1321-1329.

TARGET Consortium. (2018). Are you interested in how Augmented and Virtual Reality can be used to train Security Critical Agents? Retrieved from TARGET - Training Augmented Reality Generalised Environment Toolkit: http://www.targeth2020.eu

Webber, M., \& Rittel, H. (1973). Dilemmas in a general theory of planning. Policy Sciences, 4, 155-169. 